山्山FFRANÇAISE

$>\mathrm{DE}$

$\stackrel{1=1}{\simeq}$ PÉDAGOGIE

\section{Revue française de pédagogie}

Recherches en éducation

172 | juillet-septembre 2010

La pédagogie universitaire : un courant en plein développement

\title{
L'inéluctable ajustement des universités africaines au processus de Bologne
}

Inevitable adjustement of African universities to the Bologna Process

Ineluctable ajuste de las universidades africanas con el proceso de Bolonia

Die unausweichliche Anpassung der afrikanischen Universitäten an den

Bologna-Prozess

Jean-Émile Charlier et Sarah Croché

\section{OpenEdition}

Journals

Édition électronique

URL : http://journals.openedition.org/rfp/2276

DOI : $10.4000 /$ rfp.2276

ISSN : 2105-2913

Éditeur

ENS Éditions

Édition imprimée

Date de publication : 15 juillet 2010

Pagination : 77-84

ISBN : 978-2-7342-1188-4

ISSN : 0556-7807

Référence électronique

Jean-Émile Charlier et Sarah Croché, «L'inéluctable ajustement des universités africaines au processus de Bologne », Revue française de pédagogie [En ligne], 172 I juillet-septembre 2010, mis en ligne le 28 février 2013, consulté le 20 avril 2019. URL : http://journals.openedition.org/rfp/2276 ; DOI : $10.4000 /$ rfp.2276 


\section{Positions, débats et controverses}

\section{L'inéluctable ajustement des universités africaines au processus de Bologne Jean-Émile Charlier et Sarah Croché}

Lancé à la Sorbonne en 1998, confirmé un an plus tard dans la ville dont il a pris le nom, le processus de Bologne visait initialement à réformer l'enseignement supérieur des pays européens pour lui permettre de devenir plus attractif par rapport à celui des États-Unis. Soutenu immédiatement par la Commission européenne, que ses initiateurs avaient d'abord tenté d'écarter, le processus a rapidement connu un engouement qui a surpris tous les observateurs. L'influence de l'architecture de Bologne a dépassé les frontières de l'Europe pour toucher toutes les autres régions du monde ; elle a bien sûr atteint aussi les universités africaines. Les réformes qu'elles doivent mener pour s'aligner sur le modèle de Bologne sont considérables. Cet article cherche à identifier à la fois les raisons pour lesquelles elles peuvent trouver des avantages divers à réaliser cet alignement et l'intérêt que cela pourrait représenter pour les universités européennes d'entrer en dialogue avec elles sur les critères de qualité et les modes de connaissance.

Descripteurs (TESE) : Afrique, coopération inter-universités, enseignement supérieur, mobilité des étudiants, processus de Bologne, qualité de l'enseignement.

\section{DES OBJECTIFS NATIONAUX ET EUROPÉENS}

De nombreux analystes (Musselin, 2003 ; Racké, 2007 ; Ravinet, 2005, 2007 ; Witte, 2006) ont relevé que les ministres des quatre pays qui, en 1998, ont signé la déclaration de la Sorbonne, prélude du processus de Bologne, visaient d'abord à résoudre des problèmes qu'ils n'arrivaient pas à traiter efficacement chacun sur sa scène nationale. Sans exhaustivité ni classement des motivations des pays, il peut être rappelé que la France et l'Allemagne cherchaient à relever l'attractivité de leur enseignement supérieur, que le Royaume-Uni voulait affirmer sa position concurrentielle par rapport aux États-Unis et élever le taux de participation de ses étudiants de moins de trente ans à l'enseignement supérieur, que l'Italie et l'Allemagne entendaient réduire la durée des parcours d'études, jusque-là peu contraints, ou encore que la France voulait intégrer les grandes écoles et les universités (Charlier \& Croché, 2007 ; Croché, 2009 ; Ravinet, 2009). Chacun de ces pays avait l'intention de jouer du processus pour faire évoluer dans le sens espéré les situations nationales qui lui posaient problème. La technique est connue de longue date, elle a été analysée et théorisée par Putnam (1988) et elle a été utilisée à de multiples reprises, notamment au cours de la construction européenne (1): il est plus facile d'imposer une mesure sur la scène nationale si elle a été décidée à un palier 
international et s'applique donc à un territoire vaste, l'acceptation d'une mesure désagréable par de multiples partenaires unanimement reconnus comme raisonnables et culturellement proches tend à la rendre moins inacceptable.

Sans que cette évolution ait été recherchée ou explicitement souhaitée, le processus de Bologne a par ailleurs rendu la compréhension des mécanismes de décision plus malaisée qu'auparavant : d'une part, chaque pays est resté totalement et incontestablement maître de son enseignement supérieur alors que, d'autre part, la manière dont sont structurées les études supérieures et dont leur qualité est mesurée et garantie est décidée par la conférence des ministres réunis en sommets bisannuels, comme du reste le rythme auquel les réformes sont amenées à s'imposer dans les systèmes nationaux. II n'y a pas là de contradiction fondamentale, chaque pays qui a adhéré au processus de Bologne dispose, en la personne de son ministre responsable de l'enseignement supérieur, d'un représentant à cette conférence et il s'y exprime en pleine souveraineté. Le double palier national et de concertation internationale rend seulement les choses un peu confuses : que signifie encore la totale maîtrise de son enseignement par le ministre national, dès lors que celui-ci s'est engagé à ne prendre aucune décision qui irait à l'encontre de l'accord qu'il a passé avec tous ses collègues ? En suscitant cette confusion, le processus de Bologne a également contribué à éloigner le lieu où est prise la décision de celui où elle est censée être exécutée. Ces deux évolutions ont rendu la contestation plus difficile : le temps est révolu où il suffisait de peser sur le ministre de son pays pour qu'il modifie ses décisions politiques, la responsabilité est désormais fragmentée, les systèmes nationaux sont devenus interdépendants (même s'ils ne le sont en réalité que très faiblement), les rapports de force sont dès lors devenus plus complexes à faire évoluer, ce qui joue en faveur des décideurs en les protégeant des mouvements d'opposition ou de contestation. Cette caractéristique du processus de Bologne a largement contribué à son succès, elle n'a évidemment pas échappé aux responsables politiques qui ont conduit les universités de leur pays à y adhérer. Le processus de Bologne a donc été utilisé sous les multiples cieux de la grande Europe pour justifier des mesures nombreuses et variées, dont la majorité est objectivement indépendante de ce que ce processus impose comme ajustements (Charlier, 2008).

Dans un premier temps, le processus de Bologne ne portait en rien un projet d'européanisation des politiques d'enseignement supérieur. L'initiative de convier ses collègues du Royaume-Uni, d'Italie et d'Allemagne à la réunion de la Sorbonne sous le fallacieux (2) prétexte de fêter les huit cents ans de l'université de Paris a été prise par Claude Allègre, alors ministre français responsable de l'enseignement supérieur, qui a multiplié les déclarations les plus sévères sur l'inefficacité de la Commission européenne et sur la nécessité de laisser aux seules universités (3) le soin de conduire et de réussir le processus d'harmonisation (4) de l'enseignement supérieur. Puisque c'est en prenant appui sur cet argumentaire que Claude Allègre a obtenu le soutien de ses collègues et qu'il les a convaincus de participer à la rencontre de la Sorbonne, celle-ci peut être lue comme une tentative de préserver l'enseignement supérieur de l'emprise des instances européennes et de le maintenir dans les matières relevant exclusivement de l'autorité des États. Cette tentative n'a pas abouti et, en quelques années, la Commission s'est imposée comme un acteur majeur dans les instances du processus de Bologne, ce qui lui a permis de l'orienter pour en faire un instrument mis au service de la réussite de la stratégie de Lisbonne (Cussó, 2006). Les luttes qu'elle a menées pour prendre le contrôle du processus de Bologne l'ont amenée à la fois à évincer ses concurrents et à circonvenir les États, initialement rétifs à lui accorder davantage d'initiative dans les matières sensibles de l'enseignement supérieur et de la recherche scientifique. Les affrontements au terme desquels son leadership semble aujourd'hui unanimement admis ont été âpres et complexes. Ils ont profondément modifié les finalités du processus de Bologne et imposé de nouvelles représentations des critères auxquels doivent désormais correspondre le "bon enseignement " et la " bonne science » dans les universités européennes (Croché, 2009). Les définitions qui y sont promues de l'enseignement et de la science sont parfaitement compatibles avec celles qui sont proposées par les universités nord-américaines.

L'argument qui a fait adhérer les populations et les universités européennes au processus de Bologne est celui de la mobilité. Au-delà des rodomontades de la déclaration de la Sorbonne dans laquelle il est affirmé, contre toute vérité historique, que les universités "sont nées en Europe " (Allègre, Berlinguer, Blackstone et al., 1998), un véritable projet a été proposé, pragmatique et concret, qui visait à faire effectuer aux étudiants une partie de leur cursus dans une institution située dans un autre pays de cette " grande Europe ", dont les frontières sont délimitées par le Conseil de l'Europe. Les objectifs stratégiques du processus de Bologne, et en particulier celui de construire un enseignement supérieur européen 
capable de résister à la concurrence des États-Unis, n'ont jamais été camouflés, ils figurent dans tous les textes officiels. Cela étant, seul celui de la mobilité internationale a été réellement mobilisateur. Le programme Erasmus avait préparé les sensibilités de longue date (Papatsiba, 2001), la déclaration de la Sorbonne les a ralliées à ses objectifs. La Commission européenne ne s'y est pas trompée, elle a multiplié les communiqués triomphalistes quand la millionième bourse Erasmus a été attribuée (Commission européenne, 2002), même si seulement $1,5 \%$ des bénéficiaires potentiels avaient à ce moment reçu un soutien du programme Erasmus.

Aujourd'hui, les ambitions des autorités européennes ont été réduites. Le rapport Attali (1998), qui a largement inspiré la déclaration de la Sorbonne, au même titre que les rapports qui ont été rédigés au même moment dans les autres pays qui en ont été signataires, proposait comme objectif que la moitié des diplômés de l'enseignement supérieur effectuent une partie de leur cursus à l'étranger. En 2008, un rapport appuyé par la Commission européenne (Commission européenne, 2008) conservait cet objectif en proposant une définition on ne peut plus extensive de la mobilité, qui inclut "le passage d'une université à une autre, le passage du monde de l'éducation à celui des affaires, de l'éducation aux actions volontaires, le passage vers la formation professionnelle et vers des centres de recherche (5) ». En 2009, les ministres de l'éducation réunis à Louvain et à Louvain-la-Neuve ont opté pour l'objectif plus réaliste de faire en sorte que $20 \%$ des étudiants qui termineront leurs études supérieures en 2020 aient effectué une part de leur cursus à l'étranger (Conference of European ministers responsible for higher education, 2009). S'il tarde visiblement à se réaliser, l'objectif de faire de la mobilité une caractéristique ordinaire des parcours d'études supérieures a pourtant été massivement soutenu par les autorités publiques. Ainsi, par exemple, le programme Erasmus a été doté d'un budget de $168004652 €$ en 2004, $200962045 €$ en 2005, de $231753825 €$ en 2006 (Croché, 2006) ; la Commission européenne avait demandé 13,6 milliards d'euros comme budget 2007-2013 pour le programme Éducation et formation tout au long de la vie, qui regroupe diverses actions précédemment séparées (6), elle n'a finalement obtenu que 6,97 milliards d'euros (Parlement européen, 2009). II faut ajouter à ces montants considérables toutes les aides publiques et privées, nationales, régionales et locales et les dépenses privées des familles, de plus en plus nombreuses à être convaincues qu'un parcours d'études ne se distingue que s'il inclut un séjour à l'étranger. Cette mobili- sation impressionnante de moyens n'a pas été suffisante, jusqu'à présent, pour banaliser ce séjour, qui reste réservé à une minorité d'étudiants.

\section{UNE EXTENSION PARTIELLEMENT INCONTRÔLÉE}

Ce n'est qu'à partir du sommet de Berlin de 2003 que les ministres européens de l'enseignement supérieur ont commencé à s'intéresser explicitement à ce qu'il est convenu d'appeler la "dimension externe » du processus de Bologne, qui désigne l'ensemble des effets qu'il produit hors de l'espace européen. Avant cela, leurs sommets avaient été consacrés à la gestion des urgences actuelles, parmi lesquelles avait figuré en bonne place la résolution des problèmes de pouvoir qui ont longuement risqué de faire chavirer le processus (Croché, 2009) et la définition progressive du périmètre auquel il s'appliquerait : la candidature de l'Australie, reçue en 2001 (Charlier, Croché \& Ndoye, 2009, p. 99), avait forcé à rendre explicite ce qui avait tout d'abord été laissé imprudemment à l'arbitrage du bon sens. Cette centration sur ce qui s'est alors imposé comme l'essentiel n'a toutefois pas empêché la Commission d'être proactive: dès 2000 , elle a présenté le "modèle de Bologne " comme une référence à l'occasion de grandes réunions internationales, comme l'ont fait en parallèle I'UNESCO ou I'OCDE (Charlier \& Croché, 2003). Elle a promu l'architecture de Bologne dans son appel à projets Tempus 2000-2006, destiné à fournir à des universités des pays membres de l'Union européenne (ou qui y sont associés) les moyens d'établir des collaborations avec leurs consœurs des pays des zones TACIS (Europe de l'Est et Asie centrale), CARDS (Balkans) et MEDA (Maghreb et Proche-Orient). L'alignement sur l'architecture des études en trois cycles $(3,5$ et 8 ans) ou "l'adaptation aux développements du processus de Bologne " ont ensuite été élevés au rang d'objectifs explicites des coopérations établies avec les pays tiers (Commission européenne, 2004, 2005). Le souci de la Commission de promouvoir un modèle européen d'enseignement supérieur avait été relevé par Kehm (1997) dès avant le processus de Bologne. Le lancement de ce processus a donné une consistance concrète au modèle européen d'enseignement supérieur, qui a dès lors pu faire l'objet d'une promotion soutenue : $58 \%$ des projets Tempus de 2005 ont financé des actions d'information ou d'accompagnement visant à aider les bénéficiaires à adapter leur enseignement supérieur au « modèle de Bologne " (Commission européenne, 2005).

On ne peut que le regretter, le choix des pays européens pour l'architecture institutionnelle de Bologne 
n'a pas donné lieu à des concertations avec les pays du Sud, dont il était pourtant évident qu'ils seraient contraints de s'aligner sur le modèle universitaire européen. C'est d'autant plus regrettable que l'histoire récente avait montré que pareilles concertations étaient possibles : le passage à l'euro de la France avait été précédé d'échanges constructifs avec les pays africains dont la monnaie s'appuyait sur le franc français. D'aucuns ont voulu voir dans l'ECTS (European credit transfer system) l'équivalent de l'euro dans le monde de l'enseignement : il eût été opportun de tirer toutes les leçons de l'usage de pareille métaphore. D'une certaine manière, comme le franc CFA bénéficiait d'une parité fixe avec le franc français, de nombreux diplômes de second cycle des pays francophones d'Afrique centrale ou de l'Ouest bénéficiaient d'une parité fixe avec leurs équivalents français, ce qui permettait aux mieux défendus de leurs détenteurs de terminer leur cursus dans une université européenne et d'y acquérir le doctorat et les galons comptabilisés par le CAMES (Conseil africain et malgache pour l'enseignement supérieur). Le passage au LMD (licence, master, doctorat (7)) a la vertu de tout remettre en question. Dans la lecture positive qui peut en être faite, il libère les institutions du Sud installées dans une longue dépendance, qui peuvent désormais plus aisément conclure des accords avec d'autres universités du Nord plus respectueuses de leurs différences. Dans une lecture plus négative, il dégage les universités du Nord des accords historiques conclus avec leurs homologues du Sud, qui risquent dès lors de perdre des appuis qui leur étaient très utiles. L'observation de terrain suggère qu'aucune de ces deux lectures ne rend correctement compte de la réorganisation en train de s'opérer. Le passage au LMD contribue à ouvrir la gamme des choix possibles de partenariats, tant pour les universités du Nord que pour celles du Sud. Divers facteurs, tels la langue et la proximité culturelle entre les pays et les universités, restreignent toutefois immédiatement ce choix. En définitive, les partenariats s'établissent davantage en fonction des affinités entre les partenaires qu'en fonction d'une évaluation objectivée des besoins.

La demande de mobilité que les étudiants africains adressent aux institutions universitaires du continent européen semble pourtant intarissable. Pour présenter son Recueil mondial de données sur l'éducation de 2006, I'UNESCO avait publié un communiqué dans lequel elle affirmait sobrement que les étudiants africains étaient les "champions de la mobilité " (UNESCO, 2006), et ce titre ne peut leur être contesté. Les chiffres sont spectaculaires : un étudiant d'Afrique subsaharienne sur 16 , soit $5,6 \%$, part étudier à l'étranger, alors que ce n'est le cas que d'un étudiant nord-américain sur 250 , soit $0,4 \%$. La majorité de ces étudiants africains mobiles quittent leur continent, principalement pour aller en Europe. Ces quelques informations donnent la mesure de la confiance qu'accordent les étudiants africains aux universités établies sur leur territoire. Elles font apparaître aussi que le choix africain pour le LMD n'a rien de commun avec le choix qu'a exprimé l'Europe pour cette organisation de l'enseignement supérieur. L'Europe envisageait de renforcer la visibilité et l'attrait de son enseignement supérieur sur la scène internationale, elle souhaitait dans le même temps contribuer à doter les diplômés d'une conscience européenne en les encourageant à opérer une part de leur cursus dans un autre pays que leur pays d'origine. Ces deux objectifs fondateurs ne sont pas à l'agenda des pays africains qui vont opter pour le LMD. Les moyens qu'exigerait l'organisation d'une mobilité intraafricaine des étudiants et des personnels ne sont pas actuellement disponibles et rien n'indique qu'ils le seront prochainement. Nous avons vu que les montants mobilisés en Europe sont colossaux et que, malgré cela, ils ne parviennent à faire bouger qu'une toute petite minorité des étudiants du continent. Ces constats conduisent à la conclusion selon laquelle le transfert du modèle de Bologne vers l'Afrique est tronqué et amputé d'une partie essentielle de ce qui lui donnait du sens en Europe. Ne résiste correctement au transfert que l'appareil des normes et des conventions, qui n'avaient de sens en Europe que pour faciliter les échanges d'étudiants et soutenir l'image d'un système d'enseignement supérieur intégré.

Assurée du succès de sa stratégie de construction d'un espace européen de l'enseignement supérieur, de la recherche et de la formation tout au long de la vie, consciente des attentes et des inquiétudes que le modèle de Bologne suscite en Afrique, l'Europe tente désormais de structurer des programmes d'échanges avec les universités africaines. Elle ne s'est tournée vers elles, qui constituent ses partenaires les plus naturels, qu'après avoir recherché de manière fructueuse des collaborations avec les pays d'Asie, d'Amérique latine et du Nord. L'enjeu premier du processus de Bologne tel qu'il a été amorcé à la Sorbonne était en effet, faut-il le rappeler, d'améliorer l'attrait de l'enseignement supérieur européen dans les régions du monde où il tendait à ternir. Les discussions sur la forme que pourrait prendre les partenariats en matière d'enseignement supérieur entre l'Afrique et l'Europe n'ont pas été amorcées dans le cadre du processus de Bologne. Elles ont été au centre des discussions sur la stratégie conjointe entre 
l'Afrique et l'Union européenne lancée en 2007. Ces échanges entre des partenaires égaux ont débouché sur un premier plan d'action 2008-2010 (Conseil de I'Union européenne, 2007 ; Commission européenne, 2009b). La conférence «Developing links: EU-Africa cooperation in higher education through mobility ", qui a rassemblé en décembre 2008 des représentants d'universités africaines et européennes, des représentants de la Commission européenne, de la Commission de l'Union africaine et du secrétariat des pays d'Afrique, des Caraïbes et du Pacifique (ACP), a souligné le besoin de réformer l'enseignement supérieur en Afrique et d'établir des structures et des systèmes compatibles, qui faciliteront la mobilité (8) entre les pays d'Afrique et entre ceux-ci et l'Europe (EU-Africa cooperation in higher education, 2008). Enfin, pour la première fois, des observateurs africains (Afrique du Sud, Égypte, Éthiopie, Maroc, Sénégal, Tanzanie, Tunisie) ont figuré parmi les vingt pays non européens invités à la conférence ministérielle de suivi du processus de Bologne qui s'est tenue à Louvain et à Louvain-la-Neuve en avril 2009, au cours de laquelle ils ont pu s'exprimer sur les réformes de leur enseignement supérieur (Commission européenne, 2009a). Cette avancée s'inscrit dans l'installation d'un forum politique de Bologne en 2009, qui veut favoriser le dialogue entre les pays membres de l'Espace européen de l'enseignement supérieur et ceux des autres régions du monde. Elle a été suivie de la publication, par la Commission européenne, d'un appel à projets pour la réalisation d'une étude de faisabilité d'une approche de l'enseignement supérieur en Afrique, basée sur la méthode du projet Tuning. Développée depuis 2001 en Europe, cette méthode veut instaurer une description des différents cycles d'études et des programmes en termes de compétences.

Il est évident que les échanges massifs dans les deux sens d'étudiants entre l'Afrique et l'Europe ne font pas partie des perspectives imaginables de manière réaliste à court terme. Trop de problèmes pratiques les rendent aujourd'hui particulièrement difficiles. La pratique d'échanges réguliers n'est imaginable qu'entre des institutions dont le calendrier académique est fixé de longue date et est assez systématiquement et scrupuleusement respecté. Or, ces dernières années, la date de démarrage des enseignements dans la majorité des universités francophones d'Afrique de l'Ouest, par exemple, est impossible à prédire car elle est déterminée par le rapport de force entre les syndicats, le pouvoir politique et les bailleurs de fonds. De la même manière, il est impossible de prévoir la fin du semestre et plus encore la date de publication des notes: dans cer- tains conflits, les enseignants refusent de corriger les copies ou bloquent les notes pour faire pression sur le gouvernement. L'inscription de ces universités dans des systèmes d'échanges avec d'autres institutions, que ce soit avec des homologues africaines qui seront érigées en centres d'excellence dans certaines matières ou que ce soit avec des homologues occidentales, va paradoxalement donner davantage de puissance aux groupes contestataires. Tant qu'il n'y avait pas d'interdépendance entre les universités des différents pays, les gouvernements pouvaient sans trop de problème laisser s'enliser les conflits sociaux : la date de rentrée ou de remise des notes était indéfiniment différée sans que cela ne gêne fondamentalement les membres de l'université en grève. Dès lors que les universités seront mises en interdépendance, comme ce sera le cas quand des échanges structurés seront organisés entre elles, le blocage de l'une d'elles deviendra un problème pour l'ensemble du réseau dont elle fait partie. On peut donc s'attendre à ce que la construction des réseaux africains d'universités aille de pair, au cours des prochaines années, avec des conflits très durs qui ne pourront se résoudre que par la standardisation des conditions salariales et professionnelles des universitaires d'une même zone socio-géographique.

\section{LES UNIVERSITÉS EUROPÉENNES ONT BESOIN DU DIALOGUE AVEC LEURS HOMOLOGUES AFRICAINES}

II serait bon, tant pour les universités africaines que pour leurs homologues européennes, que les premières acceptent de s'inscrire dans le nouvel espace international qui se met en place et qu'elles y importent leurs préoccupations urgentes. Le processus de Bologne a la vertu incontestable d'avoir mis en place des mécanismes multiples qui facilitent la circulation des personnes et des savoirs entre quarante-sept pays. II a le défaut d'avoir limité cette circulation à cette petite cinquantaine de pays, ou encore d'avoir suscité la structuration de cet ensemble en lui donnant la fonction première de se mettre en concurrence (et il serait plus correct de dire en compétition) avec les deux autres ensembles que constituent les systèmes d'enseignement supérieur d'Amérique du Nord et d'Asie. Nous voulons continuer de croire que les universités valent mieux que cela et que les mettre au service de la construction de l'économie de la connaissance la plus efficace et la plus cohésive au monde revient à leur mesurer chichement notre confiance et à retarder le moment où la mobilisation des intelligences universitaires sera faite au bénéfice indistinct de tous les humains. Dans un monde globalisé, l'université ne 
trouvera ses limites que quand elle sera mise au défi de résoudre les problèmes urgents de l'humanité, quand il sera demandé à tous ceux qui la servent de se mobiliser collectivement pour leur trouver des solutions concrètes et praticables. Il s'agit désormais d'une utopie partagée, qui se lit aussi dans la position adoptée par I'UNESCO lors de la Conférence mondiale de l'enseignement supérieur de juillet 2009, dont le communiqué stipule que "l'enseignement supérieur devrait, au sein de chaque société, être la première source de connaissances mondiales sur les moyens de relever des défis mondiaux tels que la sécurité alimentaire, le changement climatique, la gestion de l'eau, le dialogue interculturel, les énergies renouvelables et la santé publique " (UNESCO, 2009). Cette citation atteste que l'utopie, exprimée à petit bruit dans de nombreux documents, dont par exemple la conclusion des actes du colloque de Dakar de 2005 (Charlier, Croché \& Ndoye, 2009), est amplifiée par l'autorité d'une organisation internationale, ce qui la promeut au statut d'élément potentiel d'un programme politique.

II ne sera possible d'envisager l'enseignement supérieur de la sorte que si les systèmes nationaux et internationaux de mesure de la qualité de l'enseignement supérieur valorisent en priorité les travaux centrés sur des questions qui concernent l'humanité tout entière et les publications qui visent à mettre leurs résultats à la disposition de chacun sans restriction aucune. Dans tous les cas, ces critères de qualité ne sont que des conventions dont la force tient à la confiance qui leur est accordée dans les univers où ils s'appliquent. Ils ne peuvent donc pas être établis en négligeant les intérêts des groupes qui sont amenés à les respecter et à s'en ériger les défenseurs. Laisser s'établir et se stabiliser ces critères sans intervenir revient à accorder une prime aux plus puissants, en l'occurrence aux organismes internationaux de certification les plus à même de concevoir rapidement tout le matériel technique d'accompagnement d'une démarche qualité et aux institutions du Nord les mieux aguerries aux négociations complexes avec les partenaires académiques et commerciaux de tous horizons. Les pays du Sud réclament des solutions pragmatiques à des questions qui empêchent leurs populations de se projeter sereinement dans l'avenir. Un des critères de qualité de l'enseignement qui y sera dispensé pourrait donc bien être la pertinence des réponses qui seront proposées à ces questions immédiates, triviales et urgentes. Cet enjeu de la qualité est tout sauf anodin. Les critères qui s'ébauchent aujourd'hui sont ajustés aux exigences des laboratoires du Nord, leur promotion favorise objectivement la préparation de professionnels d'élite capables de s'intégrer à des équipes du Nord. La réflexion sur la qualité ne peut s'abstraire des terrains où elle est menée, elle aboutit à des conclusions différenciées en fonction de ces lieux. La poursuite d'idéaux abstraits s'inscrit dans une finalité aussi légitime pour des laboratoires du Nord englobés dans des institutions couvrant tous les domaines de la connaissance que la mise au point de modes opératoires s'impose comme un but prioritaire à certains laboratoires du Sud. La relativité des critères de qualité incite à espérer qu'avant d'être décrétés universels, ils seront mis en débat et discutés avec les collègues des universités africaines inscrites dans les sociétés les plus démunies. Cela pourrait très heureusement aboutir aussi à faire admettre qu'un des critères importants de qualité de l'action d'une université du Nord est sa capacité à répondre de façon pertinente aux questions immédiates, triviales et urgentes que lui adressent ses environnements.

Le dialogue entre universités du Sud et du Nord pourrait aussi avoir pour effet positif de revivifier la réflexion sur les modes de connaissance. Un travail précis et précautionneux de repérage des questions qui se posent dans les pays du Sud et de la façon dont elles ont traditionnellement été prises en charge par les populations mériterait d'être opéré. Le rapport au monde porté par la science occidentale lui a indubitablement permis d'engranger des succès remarquables et il est hors de question de le contester aveuglément. La position hégémonique qu'il a conquise doit toutefois lui donner l'assurance nécessaire pour s'ouvrir à d'autres modes de perception et de connaissance du monde. L'acceptation de la pertinence, au moins partielle, de ces autres modes de connaissance ne va certainement pas de pair avec un renoncement aux critères épistémologiques qui fondent la science occidentale. Simplement certains savoirs indigènes ou autochtones conduisent à des conclusions qui paraissent techniquement efficaces, alors que le raisonnement qui guide vers ces conclusions demeure obscur. II convient dès lors de tenter de comprendre ces modes de connaissance et de les mettre en commun autant que faire se peut. Dans la foulée, il s'agit de se questionner à nouveaux frais sur les limites de la pertinence du modèle de connaissance que nous partageons.

Jean-Émile Charlier charlier@fucam.ac.be

Groupe de recherche sociologie action sens, facultés universitaires catholiques de Mons, Belgique

Sarah Croché

Groupe de recherche sociologie action sens,

facultés universitaires catholiques de Mons, Belgique et Fonds de la recherche scientifique, Belgique 
(1) Cette technique a été très abondamment décrite et commentée par les analystes et les théoriciens de l'intégration européenne.

(2) Nous ne pouvons ici développer en quoi ce prétexte était fallacieux. Sur cette question, comme sur un certain nombre d'autres qui ne sont ici qu'effleurées, nous renvoyons à Charlier, Croché et Ndoye (2009, p. 30 et suivantes).

(3) Parmi beaucoup d'autres citations possibles: "La Commission européenne s'occupe de choses qui n'étaient pas dans le Traité de Rome, comme l'éducation pour laquelle ce qui doit être fait est une harmonisation sur la base du volontariat " (Allègre, 2000, p. 304) ; «La Commission avait échoué parce qu'elle avait voulu unifier et donc ignorer les nations et leurs traditions. Nous voulions faire l'inverse » (Allègre, 2006, p. 122) ; « J'avais indiqué que nous refusions la tutelle de la Commission européenne et son argent, et que nous souhaitions que l'harmonisation des cursus soit construite par les universités elles-mêmes " (Allègre, 2006, p. 125).

(4) Le terme « harmonisation " avait été très soigneusement évité par le Conseil des ministres de l'Éducation des pays membres de l'Union européenne. II a été utilisé une seule fois, à la Sorbonne, ce qui a provoqué de nombreuses réactions négatives. II a disparu du vocabulaire politiquement acceptable à partir du sommet de Bologne de 1999 pour être remplacé par diverses litotes.

(5) II s'agit de notre traduction.

(6) Ces actions sont par exemple: Leonardo da Vinci, Socrates II, eLearning, Erasmus Mundus, Jean Monnet, Europass.

(7) Le LMD est l'appellation utilisée en France d'abord, dans certains pays francophones ensuite, pour signifier le découpage des études en trois cycles « 3,5 et 8 ans", aussi appelé " $\mathrm{Ba} \mathrm{Ma}$ $\mathrm{PhD}$ » ( bachelor, master, PhD ») dans certains pays de tradition anglo-saxonne.

(8) À la suite des programmes Alfa, Asia Link, Edulink, etc., qui ont permis d'établir ou d'intensifier les collaborations entre les pays d'Europe et ceux d'Amérique latine et du Nord, d'Asie et des Caraïbes et du Pacifique, la Commission européenne est à l'initiative d'un programme Nyerere-Erasmus (Commission européenne, 2009 b) qui veut permettre aux étudiants africains de parfaire leur formation en Europe et qui les engage à retourner en Afrique à la fin de leurs études.

\section{BIBLIOGRAPHIE}

ALLÈGRE C. (2000). Toute vérité est bonne à dire. Paris : Fayard et Laffont.

ALLĖGRE C. (2006). Vous avez dit matière grise ? Paris : Plon.

ALLÈGRE C., BERLINGUER L., BLACKSTONE T. \& RÜTTGERS J. (1998). Sorbonne joint declaration. Joint declaration on harmonisation of the architecture of the European higher education system. Disponible sur Internet à l'adresse <http://www.bologna-berlin2003.de/pdf/Sorbonne_ declaration.pdf> (consulté le 30 août 2009).

ATTALI J. (1998). Pour un modèle d'enseignement supérieur [rapport]. Paris: ministère de l'Éducation nationale, de la Recherche et de la Technologie. Disponible sur Internet à l'adresse : <http://media.education.gouv.fr/ file/94/9/5949.pdf> (consulté le $1^{\mathrm{er}}$ octobre 2010).

CHARLIER J.-É. (2008). «Repères pour analyser le processus de Bologne ». In S. Hanhart, A. Gorga, M.-A. Broyon \& T. Ogay, De la comparaison en éducation. Paris: L'Harmattan, p. 103-123.

CHARLIER J.-É. \& CROCHÉ S. (2003). "Le processus de Bologne, ses acteurs et leurs complices ". Éducation et sociétés, $\mathrm{n}^{\circ} 12, \mathrm{p} .13-34$.

CHARLIER J.-É. \& CROCHÉ S. (2007). « The Bologna process: The outcome of competition between Europe and the USA and now a stimulus to this competition ». European Education, vol. 39, $\mathrm{n}^{\circ}$ 4, p. 10-26.

CHARLIER J.-É., CROCHÉ S. \& NDOYE A. (2009). Les universités africaines francophones face au LMD. Louvain-la-Neuve : Academia-Bruylant.

COMMISSION EUROPÉENNE (2002). «Un million d'étudiants Erasmus ». Une réussite européenne. Bruxelles : Commission européenne. Disponible sur Internet à l'adresse : $<$ http://ec.europa.eu/education/archive/million/million_ fr.html> (consulté le 29 septembre 2009).

COMMISSION EUROPÉENNE (2004). «Communication de la Commission au Conseil, au Parlement européen, au Comité économique et social européen et au Comité des régions. Rapport annuel Tempus 2004 ». Bruxelles :
Commission européenne. Disponible sur Internet à l'adresse : <http://eacea.ec.europa.eu/tempus/doc/ report2004_fr.pdf> (consulté le 30 septembre 2010).

COMMISSION EUROPÉENNE (2005). «Tempus : modernisation de l'enseignement supérieur chez les voisins de I'UE ». Bruxelles: Commission européenne. Disponible sur Internet à l'adresse : <http://ec.europa.eu/education/external-relation-programmes/doc70_fr.htm > (consulté le 28 juin 2006).

COMMISSION EUROPÉENNE (2008). « Report of the high level expert forum on mobility. Making learning mobility an opportunity for all ». Bruxelles : Commission européenne. Disponible sur Internet à l'adresse : <http://ec.europa. eu/education/doc/2008/mobilityreport_en.pdf> (consulté le 30 septembre 2010).

COMMISSION EUROPÉENNE (2009a). «Le processus de Bologne. Réformer les universités au cours de la prochaine décennie ". Communiqué de presse, IP/09/615, 22 avril 2009. Disponible sur Internet à l'adresse: <http://europa.eu/rapid/pressReleasesAction.do?refere nce $=$ IP $/ 09 / 615 \&$ format $=H T M L \&$ aged $=0$ \&language $=F R \&$ guilanguage $=\mathrm{fr}>$ (consulté le 30 septembre 2010).

COMMISSION EUROPÉENNE (2009b). "Policy dialogue in higher education with Africa". Bruxelles: Commission européenne. Disponible sur Internet à l'adresse : $<$ http://ec.europa.eu/education/external-relation-programmes/doc1181_en.htm> (consulté le 10 octobre 2009).

CONFERENCE OF EUROPEAN MINISTERS RESPONSIBLE FOR HIGHER EDUCATION (2009). " The Bologna process 2020. The European higher education area in the new decade ". Communiqué de la Conference of European ministers responsible for higher education, Louvain et Louvain-la-Neuve, 28 et 29 avril 2009. Disponible sur Internet à l'adresse: <http://www.ond.vlaanderen.be/ hogeronderwijs/bologna/conference/documents/Leuven_Louvain-la-Neuve_Communiqu\% C 3\% A9 April_2009.pdf> (consulté le 30 septembre 2010). 
CONSEIL DE L'UNION EUROPÉENNE (2007). Déclaration de Lisbonne. Sommet UE-Afrique. Lisbonne : Conseil de l'Union européenne. Disponible sur Internet à l'adresse : <http://register.consilium.europa.eu/pdf/fr/07/st16/ st16343.fr07.pdf> (consulté le 11 octobre 2009).

CROCHÉ S. (2006). "Qui pilote le processus de Bologne?» Éducation et sociétés, $\mathrm{n}^{\circ} 18$, p. 203-217.

CROCHÉ S. (2009). Bologne confisqué. Constitution, autour de la Commission européenne, d'un acteur-réseau et d'un dispositif européen de l'enseignement supérieur. Thèse de doctorat, sciences politiques et sociales et sciences de l'éducation, facultés universitaires catholiques de Mons, Belgique et université Lumière-Lyon 2.

CUSSÓ R. (2006). « La Commission européenne et l'enseignement supérieur. Une réforme au-delà de Bologne ». Cahiers de la recherche sur l'éducation et les savoirs, $n^{\circ} 5$, p. 193-214.

EU-AFRICA COOPERATION IN HIGHER EDUCATION (2008). Developing links: EU-Africa cooperation in higher education through mobility. Bruxelles : EU-Africa cooperation in higher education. Disponible sur Internet à l'adresse : <http://ec.europa.eu/education/external-relation-programmes/doc/confafrica.pdf> (consulté le 10 octobre 2009).

KEHM B. (1997). «Evaluating the Tempus programme: Higher education development in Central and Eastern Europe ". Journal of Studies in International Education, vol. 1, $\mathrm{n}^{\circ} 45$, p. $45-56$.

MUSSELIN C. (2003). «Quelle place pour les universités françaises dans la conquête de leur autonomie : vers un nouveau modèle européen ? " Communication présentée au $14^{\mathrm{e}}$ colloque du Groupement international des secrétaires généraux des universités francophones, Paris. Disponible sur Internet à l'adresse : <http://www. gisguf.org/colloq-form/2003-paris/musselin.pdf> (consulté le 30 septembre 2010).

PAPATSIBA V. (2001). Le séjour d'études à l'étranger: formation, expériences. Analyse des rapports d'étudiants français ayant bénéficié du programme Erasmus. Thèse de doctorat, sciences de l'éducation, université de Paris-Nanterre.

PARLEMENT EUROPÉEN (2009). «Éducation et formation tout au long de la vie: programme intégré d'action incluant les programmes Comenius, Erasmus, Leonardo da Vinci et Grundtvig et le programme Jean Monnet ». Fiche de procédure, COD/2004/0153. Strasbourg:
Parlement européen. Disponible sur Internet à l'adresse : <http://www.europarl.europa.eu/oeil/FindByProcnum.do?lang=fr\&procnum=COD/2004/0153> (consulté le 28 septembre 2009).

PUTNAM R. (1988). « Diplomacy and domestic politics: The logic of two-level games ». International Organization, vol. $42, n^{\circ} 3$, p. 428-460.

RACKÉ C. (2007). " The emergence of the Bologna process ». In D. De Bièvre \& C. Neuhold (dir.), Dynamics and obstacles of European governance. Cheltenham: Edward Elgar Publishing.

RAVINET P. (2005). « The Sorbonne meeting and declaration: Actors, shared vision and europeanisation ". Communication présentée à la third conference on knowledge and politics, University of Bergen. Disponible sur Internet à l'adresse: <http://www.knowpol.uib.no/portal/ files/uplink/1312.pdf> (consulté le 11 juin 2006).

RAVINET P. (2007). La genèse et l'institutionnalisation du processus de Bologne. Entre chemin de traverse et sentier de dépendance. Thèse de doctorat, science politique, Institut d'études politiques, Paris.

RAVINET P. (2009). "Comment le processus de Bologne a-t-il commencé ? "Éducation et sociétés, $\mathrm{n}^{\circ} 24$, p. 29-44.

UNESCO (2006). "Les étudiants africains champions de la mobilité ». Communiqué de presse, $n^{\circ}$ 2006-54, 31 mai 2006. Disponible sur Internet à l'adresse : <http://portal.unesco.org/fr/ev.php-URL_ID=33154\&URL DO=DO_TOPIC\&URL_SECTION=201.html> (consulté le 28 septembre 2009).

UNESCO (2009). « Conférence mondiale sur l'enseignement supérieur 2009. La nouvelle dynamique de l'enseignement supérieur et de la recherche au service du progrès social et du développement ». Communiqué, $n^{\circ}$ ED-2009/CONF. 402/2, 8 juillet 2009. Disponible sur Internet à l'adresse : <www.iesalc.unesco.org.ve/dmdocuments/communique_cmes09fr.pdf> (consulté le 30 septembre 2010).

WITTE J. (2006). Change of degrees and degrees of change. Comparing adaptations of European higher education systems in the context of the Bologna process. Thèse de doctorat, sociologie, université de Twente, PaysBas. Disponible sur Internet à l'adresse : <http://www. che.de/downloads/C6JW144_final.pdf> (consulté le 30 septembre 2010). 